\title{
SOBRE EL ALCANCE DEL PRECOMPROMISO
}

\author{
José Juan Moreso \\ (Universidad Pompeu Fabra)
}

\section{Introducción}

En mi trabajo 'Derechos y justicia procesal imperfecta' ${ }^{1}$ presentaba una defensa de la idea con arreglo a la cual, si aceptamos una teoría de la justicia que contiene principios que establecen derechos básicos para las personas, entonces estamos comprometidos con un diseño institucional que de alguna manera atrinchere en un coto vedado esos derechos básicos. Esta idea era presentada de manera que pudiera enfrentar algunas críticas recientes: me referí no sólo, aunque fundamentalmente, a la posición de Jeremy Waldron ${ }^{2}$, sino también a la de Juan Carlos Bayón y Roberto Gargarella. Por fortuna, Gargarella ha escrito una crítica dirigida directamente a mi trabajo y Bayón ha puesto por escrito sus ideas al respecto, ideas que hasta ahora conocíamos sólo en su versión oral. ${ }^{3}$ Juan Carlos y Roberto disponen de una agudeza mental y de una preparación filosófica admirables, pero como también son mis amigos confío en que serán condescendientes y comprenderán que en estas pocas páginas no trate de presentar una concepción totalmente articulada capaz de salir al paso de sus poderosas críticas. Mi propósito es más modesto: trataré solamente de aclarar aquellos aspectos relacionados con la idea de precompromiso, una idea que a ellos no les parece valiosa para fundamentar la protección institucional del coto vedado y que yo, en cambio, considero todavía útil para este propósito. Terminaré con unas reflexiones más generales acerca de la idea de primacía de la Constitución y de la justificación del control de constitucionalidad.

\section{Acerca del precompromiso}

Las críticas que Bayón y Gargarella dirigen al mecanismo del precompromiso entendido como una explicación y como una justificación 
de aquellos diseños institucionales que atrincheran determinados aspectos para situarlos más allá de la decisión mayoritaria pueden, tal vez, ser agrupadas en las dos siguientes:

1) El problema de la identidad: El problema surge de una analogía entre el plano individual y el plano colectivo que, se arguye, es engañosa. La sociedad no es la misma a lo largo del tiempo y la sociedad no tiene una opinión sino muchas opiniones. Obviamente que yo no creo que la sociedad sea ninguna entidad orgánica ni nada parecido. Ahora bien, existe en esta crítica el presupuesto de que la identidad de los agentes individuales no es problemática y que un agente individual tiene en cada momento una sola opinión acerca de cómo debe comportarse. Es paradójico que los autores que se han ocupado del problema de la identidad individual pongan en duda precisamente este presupuesto y sostengan que el concepto de identidad individual ha de construirse mediante una analogía con el concepto de identidad de una sociedad. Hume, por ejemplo, afirmaba: ${ }^{4}$

I cannot compare the soul more properly to any thing than to a republic or commonwealth, in which the several members are united by the reciprocal ties of government and subordination, and give rise to other persons, which propagate the same republic in the incessant changes of its parts.

Es más, de hecho se ha sugerido que una mejor comprensión de los mecanismos de decisión racional individual se alcanzará si vemos las decisiones individuales como decisiones colectivas. T.C. Schelling ${ }^{5}$ por ejemplo, convencido por trabajos como el de K. Arrow ${ }^{6}$ de que es inútil moldear la decisión colectiva sobre nuestra teoría de la racionalidad individual, puesto que -como se sabe- las decisiones mayoritarias (dado que la unanimidad no suele estar a nuestro alcance) no reflejan siempre preferencias colectivas racionales ${ }^{7}$; ha sostenido que debemos aceptar la sugerencia de que los seres humanos no somos individuos racionales individuales, sino que nos parecemos más a pequeñas colectividades con sus propios conflictos. Así, por ejemplo, Susan Hurley ha desarrollado 
una sugestiva teoría de la deliberación racional con el supuesto de que las formas de resolver los conflictos entre varias personas pueden servir como una vía para explorar la solución a los problemas de conflicto en la deliberación individual. ${ }^{8}$

En conclusión, sin entrar en el espinoso problema filosófico de la identidad, puede argumentarse que la deliberación y los mecanismos de decisión individual guardan mucha semejanza con la deliberación y los mecanismos de decisión colectiva. Dado que la pluralidad y el conflicto no sólo son un problema para las decisiones colectivas, sino también para las decisiones individuales; entonces el mecanismo del precompromiso no puede impugnase en el caso de las primeras y justificarse en el caso de las segundas. La fortuna del mecanismo del precompromiso como un instrumento de alcanzar decisiones racionales depende de su capacidad, tanto en las decisiones individuales como en las colectivas, de mediar de manera consistente y razonable con la pluralidad y el conflicto.

2) Tanto Bayón como Gargarella señalan acertadamente que, en los casos como el de Ulises, el precompromiso está justificado porque se supone que el yo que ata a su yo sucesivo es un agente con mayor lucidez, con mayor calidad deliberativa, una especie de Ulises sobrio atando las manos a los posibles Ulises ebrios. Y añaden que, en los casos de decisiones colectivas, no puede presuponerse que en los momentos constitucionales se tomen decisiones más lúcidas que en los momentos de política ordinaria (diciéndolo con la terminología de Ackerman) ${ }^{9}$.

Quiero aclarar de entrada que el mecanismo del precompromiso es sólo un instrumento. El precompromiso tiende a asegurar decisiones acertadas en la medida en que lo que el mecanismo protege es algo digno de protegerse. Ello ocurre tanto en las decisiones individuales como en las decisiones colectivas. Así, la persona que ordenara a su banco que retiren cada mes de sus ingresos una cantidad determinada y la entreguen a un comercio de bebidas alcohólicas para que éste le suministre una botella de whisky al día con el fin de consumirla él sólo por la noche, estaría atándose las manos por un objetivo que, la mayoría de nosotros, 
consideramos disvalioso. De la misma forma, si una Constitución contuviera una disposición conforme a la cual determinados delitos deben ser castigados siempre con la pena de muerte, este mecanismo de precompromiso atrincheraría una decisión que, muchos de nosotros, consideramos inmoral. La calidad de una decisión constitucional no depende de que esté atrincherada sino de su acuerdo con los principios de justicia.

Por otra parte, pueden distinguirse dos sentidos en que el mecanismo de precompromiso puede ser útil y que requieren distintas justificaciones:

a) El precompromiso como mecanismo para alcanzar resultados adecuados: La teoría de la racionalidad aplicada a los agentes individuales contempla, a menudo, el precompromiso como un mecanismo que trata de evitar que un yo futuro, ante problemas como el producido por la debilidad de voluntad u otros, frustre los deseos de alcanzar determinados resultados del yo actual. En este supuesto se precisa ciertamente que la decisión del yo actual vaya precedida de mayor calidad deliberativa que la del yo futuro al que se pretende controlar. Así nos parece que ocurre en el supuesto de la persona que prevé una velada en la que beberá una cantidad de vino que dificultará su conducción y entrega las llaves de su coche a un amigo abstemio. Entonces, es necesario que, en los casos de decisiones colectivas, mostremos que las decisiones constitucionales van precedidas de mayor calidad deliberativa que las decisiones de política normal. Obviamente que los procesos constituyentes son distintos en cada país y cada época y su calidad deliberativa es una cuestión contingente. Pero, tal vez, la mayor calidad deliberativa no haya que buscarla en cómo se toma la decisión, sino en sobre qué se toma la decisión. Habitualmente, las decisiones constitucionales reflejan acuerdos sobre cuestiones genéricas, sobre conceptos y no sobre concepciones. Es plausible pensar que, al menos, sobre algunas de estas cuestiones haya un acuerdo muy amplio. Así, en sociedades como la española, hay un acuerdo amplio sobre la adecuación de preservar la libertad religiosa. Este acuerdo puede verse sustantivamente reducido, si preguntamos sobre la adecuación de prohibir las actividades de una determinada confesión religiosa que induce 
con su doctrina y predicación al suicidio a sus miembros. Con todo, atrincherar la libertad religiosa puede servir como muralla para evitar que una mayoría (debilitada su lucidez por lo que ocurre con las actividades de dicha confesión) legisle prohibiendo las actividades no sólo de la confesión que induce al suicidio a sus miembros (lo que, en determinadas condiciones, me parece razonable), sino de todas aquellas confesiones semejantes a la primera en algunos aspectos (aunque no induzcan al suicidio).

Lo que me lleva a otra cuestión conectada con la anterior, a menudo Bayón y Gargarella (y también Waldron) argumentan como si todas las cuestiones acerca de los derechos básicos fueran igualmente controvertidas en nuestras sociedades. Si ello fuera así, es obvio que no habría forma de articular una teoría de la justicia que sirviera como fundamento de nuestro diseño institucional. Al no haber acuerdo sobre nada, la regla de la mayoría sería el único instrumento para preservar los derechos de participación de todos. Sin embargo, si no hubiera acuerdo sobre nada, no sé porque debería haber acuerdo sobre que todos deben tener derecho a participar. Y si, en cambio, se reconoce que hay acuerdo en el derecho de todos a participar (un acuerdo genérico, tampoco hay acuerdo en los detalles) no veo porqué no se reconoce que también hay acuerdo en otras cuestiones. ${ }^{10}$

b) El precompromiso como mecanismo de evitación: Algunas veces el mecanismo del precompromiso puede usarse no con la intención de alcanzar resultados adecuados, sino con la intención de evitar los peligros de un exceso de deliberación o de una deliberación muy conflictiva. Mientras el precompromiso como mecanismo para alcanzar resultados adecuados parte de la idea que se prefiere que suceda $A$ a que suceda no-A y se trata de evitar que un yo futuro elija no-A, el precompromiso como mecanismo de evitación puede ser indiferente entre varias alternativas, pero se intenta que una de ellas prevalga sobre las otras para evitar un exceso de deliberación y de conflicto. Así, por ejemplo, ante la necesidad de lavar la ropa en la lavadora una vez a la semana, un agente puede elegir entre muchas alternativas, pero puede ser razonable hacer que una de ellas se convierta en un curso de acción sobresaliente: 
tal vez destinar a ello los sábados por la mañana. Una vez tomada esta decisión, el agente puede precomprometerse -e.g. reservando todos los sábados por la mañana de su agenda o de cualquier otra manera- con el fin de evitarse los costes de deliberación semanales acerca de cuándo sería mejor lavar su ropa.

Me parece que en las decisiones colectivas pueden darse situaciones semejantes. Por ejemplo, la Constitución española (en el art. 68.1) establece que el Congreso de los Diputados estará compuesto por un mínimo de 300 y un máximo de 400 Diputados, no hay -creo- un número de Diputados que podamos considerar más adecuado; pero apartar esta cuestión de la agenda política normal puede justificarse en el deseo de impedir que la confrontación legislativa se extienda en luchas estériles para los intereses de los ciudadanos. Tal vez, así pueda también justificarse la constitucionalización de un sistema electoral determinado (mayoritario o proporcional) y algunos otros aspectos de la estructura institucional. ${ }^{11}$

En resumen, tanto el precompromiso como mecanismo para alcanzar resultados adecuados (en el caso de las decisiones colectivas, para alcanzar resultados de acuerdo con los principios de justicia), como el precompromiso como mecanismo de evitación consiguen apartar determinadas cuestiones de la agenda política cotidiana, pero es importante distinguir entre ellos porque las razones que los justifican son diferentes. $^{12}$

\section{Primacía de la Constitución, Control de Constitucionalidad y deferencia al legislador}

Terminaré planteando tres cuestiones (lexicográficamente ordenadas, en el sentido siguiente: sólo una respuesta afirmativa a la anterior otorga sentido a la siguiente), con la esperanza de que esta forma de presentar la cuestión esclarezca la zona tanto de acuerdo como de desacuerdo entre las posiciones de Bayón y Gargarella y la mía propia: 
1) ¿Está justificada la idea de primacía de la Constitución, esto es, la idea de atrincherar determinadas cuestiones para impedir que puedan ser modificadas por la regla de la mayoría?

2) ¿Está justificado establecer un mecanismo de control jurisdiccional de la constitucionalidad de las leyes ordinarias?

3) ¿Qué grado de deferencia han de tener estos órganos jurisdiccionales ante las decisiones legislativas?

1) Como resultará obvio mi respuesta a la primera cuestión es afirmativa. Sin embargo, creo que -a diferencia de una defensa absoluta del modelo de Westminster como la de Waldron-, también Bayón y Gargarella responderían afirmativamente a esta cuestión. Bayón arguye ahora en defensa de lo que denomina 'constitucionalismo débil'. Se trata de un modelo que reconoce a los procedimientos democráticos (de regla de la mayoría) mayor valor intrínseco que a cualesquiera otros, pero que argumenta que este valor intrínseco debe hacerse compatible con su valor instrumental: la mayor o menor probabilidad de alcanzar resultados justos. Un modelo como el existente en países como Canadá y Suecia, modelos constitucionales de rigidez muy leve; pero, al fin y al cabo, modelos que reconocen la primacía de la Constitución. Algo semejante ocurre con Gargarella, en su opinión el ideal de democracia deliberativa por el que se inclina no es incompatible con técnicas constitucionales como la canadiense. $^{13}$

2) A lo que Bayón y Gargarella se oponen claramente es al establecimiento de un control jursidiccional de constitucionalidad. Ambos argumentan que confiar en los jueces el control de cuándo se ha violado el coto vedado constitucionalmente protegido, es contrario a nuestros ideales democráticos. Básicamente creen que es así porque dado que las declaraciones de derechos son establecidas por principios extraordinariamente abiertos, entonces queda expedita lo que Gargarella ha denominado 'la brecha interpretativa', ${ }^{14}$ son los jueces los que acaban 
determinando cuál es el alcance de los derechos y no los ciudadanos o sus representantes. Bayón añade, en este punto, otra crítica a la idea de precompromiso: esto no es atarse las manos uno mismo, sino ponerse en manos del juicio de otros. Antes de nada quiero reconocer los límites del precompromiso también en el ámbito de la racionalidad individual: puede que una persona, previendo que va a beber en exceso, entregue las llaves a su amigo abstemio, puede ser que esta persona acabe bebiendo muy poco esa noche y que su amigo abandone esa noche su ancestral sobriedad y se resista a devolverle las llaves del coche, si esto ocurre el mecanismo fracasa estrepitosamente (tal vez, algunos ejemplos de control de constitucionalidad por los Tribunales ejemplifiquen casos de este tipo en el nivel de la racionalidad colectiva: El Tribunal Supremo de los Estados Unidos en Lochner vs. New York, por ejemplo). Casi nunca el mecanismo del precompromiso es un mecanismo causal totalmente independiente de la voluntad (tampoco en la racionalidad individual: un ejemplo clásico como es el de situar el despertador en la habitación contigua, siempre puede hallar una persona que se levante lo desconecte y vuelva a la cama para seguir durmiendo), es sólo un mecanismo que, normalmente, tiene éxito, que aumenta las probabilidades de alcanzar el éxito en circunstancias normales. Por eso, yo sostendría que la segunda pregunta debe tener una respuesta más matizada que la primera: la adecuación del control de constitucionalidad depende de circunstancias contingentes, de la cultura política a la que ese mecanismo se incorpora, por ejemplo.

Un último comentario, antes de pasar a la tercera cuestión, referido a lo que Bayón denomina 'procedimiento de determinación'. Es cierto que, habitualmente, los derechos son establecidos en forma de principios y que sus límites son imprecisos. Como Bayón nos recuerda, no es igual prohibirle al legislador que establezca la pena de muerte que prohibirle que establezca penas que comporten tratos inhumanos y degradantes. Por esta razón, como es sabido, Kelsen defendía una Constitución de detalle (no de principios), que evitara la excesiva discrecionalidad del órgano de control. ${ }^{15}$ Creo, sin embargo, que la incuestionable naturaleza abierta de los principios no conlleva que, en todos los casos, el proceso de determinación sea controvertido. Ello significaría que los conceptos contenidos en los principios no tienen casos claros de aplicación. Por el 
contrario, creo que incluso los denominados 'conceptos esencialmente controvertidos' disponen de casos de aplicación clara, disponen de paradigmas que ejemplifican su uso (aunque los criterios de uso sean controvertidos). Excelente jugador de fútbol, soneto profundo o pintura metafísica son, seguramente, buenos candidatos a conceptos esencialmente controvertidos y los criterios de uso de estos conceptos son múltiples y disputados. Sin embargo, las concepciones que no atribuyan la cualidad de buen jugador de fútbol a Pelé, o de soneto profundo a alguno de los sonetos de Shakespeare, o de pintura metafísica a alguno de los cuadros de Giorgio de Chirico, pueden ser tildadas de concepciones equivocadas. Por lo tanto, el necesario procedimiento de determinación no conlleva que todos los casos queden en manos de la discrecionalidad de los jueces (aunque, es obvio, los jueces pueden equivocarse). Parafraseando a Frege, podemos decir que un concepto esencialmente controvertido sin casos claros de aplicación no es un concepto. ${ }^{16}$

3) Si suponemos que en algunas culturas políticas, el mecanismo de control de constitucionalidad puede expresar adecuadamente el compromiso entre el valor intrínseco de los procedimientos y el valor instrumental de los procedimientos, todavía queda por resolver la cuestión de cuál debe ser el grado de deferencia del órgano encargado del control hacia el legislador, dicho de otro modo cuál debe ser el grado de presunción de constitucionalidad de las decisiones legislativas. ${ }^{17}$ Insistiría en este punto en distinguir entre casos claros y casos difíciles -aunque acepto que la distinción puede ser gradual-. Lo diré con una especie de fórmula susceptible de relacionar la claridad de un caso con la deferencia: la deferencia del órgano jurisdiccional de control de la constitucionalidad debe ir en relación inversa a la claridad del caso enjuiciado.

Acabaré con una reflexión que ya se halla en mi texto, pero sobre la que deseo insistir: a menudo los órganos jurisdiccionales de control de constitucionalidad no deciden contramayoritariamente, sino que tratan de determinar cuál debe ser el derecho vencedor en un caso de conflicto de derechos constitucionales (así ocurre muy a menudo con el recurso de amparo en sistemas constitucionales como el español). Esta es una 
función claramente jurisdiccional y sea el caso fácil o difícil no tienen ninguna decisión a la que mostrar deferencia (como no fuere a las suyas anteriores). Tomar conciencia de la importancia de esta cuestión y, por lo tanto, de la importancia de este aspecto del control de constitucionalidad -subrayado por muchos ${ }^{18}$ me parece una cuestión muy relevante. Sin embargo, mi propia posición al respecto deberá aguardar a otra ocasión. 


\section{Notas}

${ }^{1}$ En este mismo volumen.

2 Jeremy Waldron, 'A Right-Based Critique of Constitutional Rights' en Oxford Journal of Legal Studies, 13 (1993), 18-51.

${ }^{3}$ Ambas contribuciones aparecen también en este número. Aunque en un contexto de discusión diferente a éste, Bayón había presentado algunas de sus ideas en Juan Carlos Bayón, 'Principios y reglas: legislación y jurisidicción en el Estado constitucional' en Jueces para la democracia, 27 (noviembre/1996),41-49.

4 David Hume, A Treatise of Human Nature, [1739-1740], ed. L.A. Selby -Bigge, Oxford: Oxford University Press, 19782, Book I, Part IV, Section VI, p. 261. También Derek Parfit, Reasons and Persons, Oxford: Oxford University Press, 1984, pp. 209-217 con especial referencia a la analogía de Hume.

${ }^{5}$ Thomas C. Schelling, 'Ethics, Law and the Exercise of Self-Command' en Choice and Consequence. Perspectives of An Errant Economist, Cambridge, Mass.; Harvard University Press, p. 93.

${ }^{6}$ Kenneth J. Arrow, Social Cohice and Individual Values, New Haven: Yale University Press, 1963.

${ }^{7}$ Por cierto, aunque no puedo detenerme en ello aquí, frente a problemas como el teorema de imposibilidad de Arrow o la paradoja liberal de Sen (Amartya Sen, 'The Impossibility of Paretian Liberal' en Journal of Political Economy, 78 (1970), 99.152-157 y Collective Choice and Social Welfare, San Francisco, Holden-Day, 1979, cap. VI y también Harmut Kliemt, 'Acerca de la coherencia de un liberalismo individualista estricto' en Ernesto Garzón Valdés (ed.), Derecho y filosofía, Barcelona: Alfa, 1985) el modelo de democracia-regla de la mayoría sin restricciones carece, al parecer, de soluciones. En cambio, la idea de primacía de la Constitución y de atrincheramiento de un coto vedado puede tal vez constituir un inicio de salida al problema. Para esta idea aplicada a la resolución de la paradoja liberal vd. Ricardo Caracciolo, 'Derecho y decisiones colectivas' en Revista del Centro de Estudios Constitucionales, 10 (1991), 25-48.

${ }^{8}$ Susan Hurley, Natural Reasons, Oxford: Oxford University Press, 1989, pp. 226-231.

${ }^{9} \mathrm{Vd}$. Bruce Ackerman, 'The Storrs Lectures: Discovering the Constitution' en Yale Law Journal, 93 (1984), p. 1029.

${ }^{10}$ Esta cuestión nos llevaría, en último término, al problema del conocimiento moral. No puedo detenerme aquí en esta importante cuestión filosófica pero deseo añadir que posiciones antirrealistas en materia moral no son necesariamente incompatibles con la admisión de la posibilidad de cierto conocimiento en materia moral. Un conocimiento que pueden proporcionar aquellos juicios morales que contienen conceptos densos (thick concepts), conceptos como los expresados por términos como 'cruel', 'degradante', etc. que son los que aparecen en las declaraciones de derechos atrincheradas constitucionalmente y no conceptos ligeros (thin concepts), como los expresados por términos como 'bueno' o 'correcto'. Vd. para este punto, el cap. 8 de Bernard Williams, Ethics and the Limits of Philosophy, London, Fontana Press, 1985.

${ }^{11}$ Quizás así pueda también justificarse la decisión de que la Constitución deje en manos de un Banco Central determinados aspectos de la política monetaria. Creo que este tipo 
de precompromiso es el que tiene en mente Stephen Holmes en 'Gag Rules or the Politics of Omission' en Jon Elster y Rune Slagstad (eds.), Constitutionalism and Democracy, Cambridge, Cambridge University Press, 1988, pp. 19-58. Sin embargo, este mecanismo tiene límites - no está justificado usarlo cuando las alternativas no son indiferentes, porque entonces no puede elegirse cualquiera de ellas, la política de la omisión no debe transformarse en la política de la injusticia-. Me parece que algunos de los ejemplos de Holmes traspasan este límite (así cuando justifica de este modo determinada autocensura que la Cámara de Representantes de los Estados Unidos se impuso a sí misma en 1836 acerca de la esclavitud). En la discusión que siguió a mi exposición (en San Remo, junio de 1997) de 'Derechos y democracia procesal imperfecta' yo mismo traspasé este límite: puse como ejemplo de mecanismo de evitación (de evitación de una polémica entre Monarquía y República, que había atenazado la política española por más de cien años), la constitucionalización de la Monarquía en el texto constitucional de 1978. Francisco Laporta me hizo dar cuenta de que el ejemplo no era bueno: presuponía algo más que discutible, que la Monarquía y la República constituyen alternativas indiferentes.

${ }^{12}$ Curiosamente los dos tipos de precompromiso que se consideran justificados, guardan cierta analogía con los dos argumentos que, según Raz, justifican la autoridad: el argumento de la pericia (expertise) y el argumento de la coordinación. Vease Joseph Raz, 'The Justification of Authority' en The Morality of Freedom, Oxford: Oxford University Press, 1986, pp. 38-69 y una clara exposición de estos argumentos, una presentación de la discusión que han generado y una evaluación crítica de ellos en Juan Carlos Bayón, La normatividad del Derecho: deber jurídico y razones para la acción, Madrid, Centro de Estudios Constitucionales, 1991,pp. 645-691. Desafortunadamente no puedo aquí perseguir las consecuencias conceptuales de esta analogía.

${ }^{13}$ Véanse, caps. 6 y 7 de Roberto Gargarella, La justicia frente al gobierno. Sobre el carácter contramayoritario del poder judicial, Barcelona, Ariel, 1996. Deseo añadir, sin embargo, que uno de los argumentos de Gargarella -siguiendo a Carlos S. Nino, Fundamentos de Derecho Constitucional, Buenos Aires, Astrea, 1992, pp. 697-700-acerca de los límites de la legitimidad democrática (y de las decisiones justificadas por la regla de la mayoría) me produce perplejidad: se trata del límite fijado por la moral privada autorreferente. Pues, como el mismo Gargarella reconoce, una de las cuestiones más controvertidas en nuestra cultura política es la de la distinción entre moral privada y moral pública. Gargarella (p. 168) sostiene: 'La idea, en principio, sería que en las cuestiones de moral privada... ni las autoridades políticas ni los jueces deben tener poder de injerencia'. Aquí volvemos a estar como al principio: hay un coto vedado (el de la moral privada), pero ¿quién lo protege?

${ }^{14}$ Roberto Gargarella, cit. En nota 13, p. 59 y ss.

${ }^{15}$ Vd. Hans Kelsen, ¿Quién debe ser el defensor de la Constitución? [1931], trad. de R.J. Brie, Madrid, Tecnos, 1925, pp. 34-37 y nota 11.

${ }^{16}$ La frase de Frege es mucho más estricta de lo que ahora -que todos somos más o menos wittgenstenianos- estamos dispuestos a aceptar: 'Un concepto que no está precisamente definido es erróneamente denominado un concepto'. Gottlob Frege, Selections from the Philosophical Writings of Gottlob Frege, trad. de P. Geach y M. Black, Oxford, Basil Blackwell, 1970, p. 150. 
17 Este es el tema que aborda Víctor Ferrerres Comella en Justicia constitucional y democracia, Madrid, Centro de Estudios Políticos y Constitucionales, 1997. En el capítulo 6 de este libro el lector encontrará interesantes reflexiones sobre la graduación de la fuerza de la presunción de constitucionalidad de las decisiones legislativas.

${ }^{18} \mathrm{Vd}$., e.g., Robert Alexy, Teoría de los derechos fundamentales, [1986], trad. de E. Garzón Valdés, Madrid, Centro de Estudios Constitucionales, 1993, pp. 89-97. 


\section{BIBLIOGRAFÍA}

ACKERMAN, BRUCE A:

$\Uparrow$ 'The Storrs Lectures: Discovering the Constitution' en Yale Law Journal, 93 (1984)

$\Uparrow$ 'Beyond Carolene Products' en Harvard Law Review, 98 (1985), 713-746.

$\Uparrow \quad$ 'Constitutional Politics/Constitutional Law' en Yale Law Journal, 99 (1989).

$\Uparrow \quad$ We The People. Vol. I: Foundations (Cambridge, Mass., Harvard University Press, 1991)

ACKERMAN, BRUCE Y ROSENKRANTZ, CARLOS:

$\Uparrow \quad$ 'Tres concepciones de la democracia constitucional' en Fundamentos y alcance del control judicial de constitucionalidad (Madrid: Centro de Estudios Constitucionales, 1991)

\section{ALEXY, ROBERT:}

^ Teoría de los derechos fundamentales, trad. de E. Garzón Valdés (Madrid: Centro de Estudios Constitucionales, 1993)

\section{ALLAN, JAMES:}

$\Uparrow \quad$ 'Bill of Rights and Judicial Power- A Liberal's Quandary' en Oxford Journal of Legal Studies, 16 (1996), 337-352.

\section{ARROW, KENNETH J.:}

$\Uparrow \quad$ Social Choice and Individual Values (New Haven: Yale University Press, 1963) 
BARRY, BRIAN:

^ 'Is Democracy Special?’, en Peter Laslett y James Fishkin (1979), 155-196.

BAYÓN, JUAN CARLOS:

$\Uparrow \quad$ La normatividad del Derecho: deber jurídico y razones para la acción (Madrid: Centro de Estudios Constitucionales, 1991)

$\Uparrow$ 'Principios y reglas: legislación y jurisdicción en el Estado constitucional' en Jueces para la democracia, 27 (noviembre/ 1996), 41-49.

$\Uparrow$ 'Derechos, democracia y constitución' Manuscrito. Ponencia inédita presentada en una conferencia el 26 de agosto de 1996 en la Universidad de Palermo, Buenos Aires (Argentina)

\section{BEITZ, CHARLES R:}

$\Uparrow \quad$ Political Equality. An Essay in Democratic Theory, (Princeton, NJ: Princeton University Press, 1989).

\section{BENTHAM, JEREMY:}

$\Uparrow \quad$ 'Anarchical Fallacies', en The Works of Jeremy Bentham, vol. II, John Bowring (ed.) (Edinburgo: William Tait, 1838-1843)

$\Uparrow \quad$ 'Pannomial Fragments' en Jeremy Bentham, The Works of Jeremy Bentham, vol. III, John Bowring (ed.) (Edinburgo: William Tait, 1838-1843)

$\Uparrow \quad$ 'Supply without Burden; or Escheat vice Taxation' en Jeremy Bentham, Economic Writings, vol. I, William Stark (ed.) (Londres: George Allen \& Unwin, 1952-1954)

BICKEL, ALEXANDER M.:

$\Uparrow \quad$ The Least Dangerous Branch (New Haven: Yale University Press, 1962)

BLACKSTONE, WILLIAM:

$\Uparrow \quad$ Commentaries on the Laws of England, 3 vol. (Londres, Dawsons of Pall Mall, ([1765-1769] 1966). 
BULYGIN, EUGENIO:

$\Uparrow \quad$ 'Sobre el status ontológico de los derechos humanos' en Doxa, 4 (1987)

BURKE, EDMUND:

$\Uparrow$ Reflections on the Revolution in France, Conor C. O'Brien (ed.) (Harmondsworth: Penguin,1969)

BUTLER, ANDREW S.:

$\Uparrow \quad$ 'The Bill of Rights Debate: Why the New Zealand Bill of Rights Act 1990 is a Bad Model for Britain' en Oxford Journal of Legal Studies, 17 (1997), 323345..

CARACCIOLO, RICARDO:

$\Uparrow \quad$ 'Derecho y decisiones colectivas' en Revista del Centro de Estudios Constitucionales, 10 (1991)

\section{DAHL, ROBERT A.:}

$\Uparrow \quad$ A Preface to Democratic Theory, (Chicago: University of Chicago Press, 1956).

$\Uparrow$ Democracy and Its Critics (New Haven: Yale University Press, 1989)

\section{DWORKIN, RONALD:}

$\Uparrow \quad$ Taking Rights Seriously (Londres: Duckworth, 1977).

$\Uparrow$ A Matter of Principle (Cambridge, Mass.: Harvard University Press, 1985).

$\Uparrow$ Law's Empire (Cambridge, Mass., Harvard University Press, 1986)

$\Uparrow \quad$ A Bill of Rights for Britain (Londres, Chatto \& Windus, 1990).

$\Uparrow \quad$ Life's Dominion (Londres, Harper Collins, 1993)

$\Uparrow \quad$ Freedom's Law. The Moral Reading of the American Constitution (Cambridge, Mass: Harvard University Press, 1996) 
ELSTER, JON:

$\Uparrow \quad$ Ulysses and the Sirens: Studies In Rationality and Irrationality (Cambridge: Cambridge University Press, 1979)

ELY, JOHN H.:

$\Uparrow \quad$ Democracy and Distrust. A Theory of Judicial Review (Cambridge, Mass.: Harvard University Press, 1980)

FERRAJOLI, LUIGI:

$\Uparrow$ Diritto e ragione. Teoria del garantismo penale, $2^{\text {a }}$ edición revisada (Roma/Bari: Laterza, 1990)

FERRERES COMELLA, VÍCTOR:

$\Uparrow$ Justicia constitucional y democracia (Madrid: Centro de Estudios Políticos y Constitucionales, 1997)

FISHKIN, JAMES:

$\Uparrow \quad$ "Tyranny and Democratic Theory", en Peter Laslett y James Fishkin, Philosophy, Politics and Society. 5th series (Oxford: Blackwell, 1979)

FREEMAN, SAMUEL:

$\Uparrow \quad$ Constitutional Democracy and the Legitimacy of Judicial Review', Law and Philosophy, 9 (1990)..

FREGE, GOTTLOB:

$\Uparrow \quad$ Selections from the Philosophical Writings of Gottlob Frege, trad. de P. Geach y M. Black, (Oxford: Basil Blackwell, 1970)

GALLIE, W. .B:

^ 'Essentially Contested Concepts' en Proceedings of Aristotelian Society, 56 (19551956) 


\section{GARGARELLA, ROBERTO:}

^ La justicia frente al gobierno. Sobre el carácter contramayoritario del poder judicial, (Barcelona: Ariel, 1996)

\section{GARZON VALDES, ERNESTO:}

^ 'Representación y democracia' en Ernesto Garzón Valdés, Derecho, Ética y Política, (Madrid: Centro de Estudios Constitucionales, 1993)

\section{GAUS, GERALD F.:}

$\Uparrow \quad$ Justificatory Liberalism. An Essay on Epistemology and Political Theory (Oxford/New York:, Oxford University Press, 1996).

\section{GUASTINI, RICARDO:}

$\Uparrow \quad$ 'Diritti' en Distinguendo. Studi di teoria e metateoria del diritto (Torino: Giappichelli,1996)

GUTMANN, AMY y THOMPSON, DENNIS:

$\Uparrow \quad$ 'Moral Disagreement in a Democracy', Social Philosophy and Policy, 12 (1995)

\section{HART, HERBERT L. A.:}

$\Uparrow \quad$ The Concept of Law (Oxford: Oxford University Press, 1961)

$\Uparrow \quad$ 'Natural Rights: Bentham and John Stuart Mill' en Essays on Bentham. Jurisprudence and Political Theory (Oxford: Oxford University Press, 1982)

$\Uparrow \quad$ 'Are there Any Natural Rights' en Jeremy Waldron, Theories of Rights, Jeremy Waldrom, editor (Oxford: Oxford University Press, 1984)

$\Uparrow \quad$ The Concept of Law, 2nd. ed., P.A. Bulloch and J. Raz (Oxford: Oxford University Press, 1994)

\section{HOHFELD, WESLEY N:}

$\Uparrow \quad$ Fundamental Legal Conceptions as Applied in Judicial Reasoning (New 
Haven: Yale University Press, 1919)

\section{HOLMES, STEPHEN:}

$\Uparrow \quad$ 'Precomittment and the Paradox of Democracy' en Jon Elster y Rune Slagstad (eds.), Constituionalism and Democracy (Cambridge: Cambridge University Press, 1988)

$\Uparrow \quad$ Passions and Constraint. On the Theory of Liberal Democracy (Chicago: University of Chicago Press, 1995)

$\Uparrow \quad$ 'Gag Rules or the Politics of Omission' en Jon Elster y Rune Slagstad (eds.), Constitutionalism and Democracy (Cambridge: Cambridge University Press, 1988)

HUME, DAVID:

$\Uparrow \quad$ A Treatise of Human Nature, L.A. Selby-Bigge (ed.) (Oxford: Oxford University Press, 1978)

\section{HUTCHINSON, ALLAN C.:}

$\Uparrow \quad$ Waiting for CORAF. A Critique of Law and Rights (Toronto: University of Toronto Press, 1995)

\section{HURLEY, SUSAN:}

$\Uparrow \quad$ Natural Reasons (Oxford: Oxford University Press, 1989).

KANT, IMMANUEL:

$\Uparrow \quad$ La metafísica de las costumbres, trad. de A. Cortina Ors y J. Conill Sancho (Madrid: Tecnos, 1989)

KELSEN, HANS:

^ ([1928] 1988): «La garantía jurisdiccional de la Constitución (la justicia constitucional)», en $\mathrm{H}$. Kelsen, Escritos sobre la democracia y el socialismo, selección y presentación a cargo de J. Ruiz Manero, Madrid, 
Debate, pp. 109-155 [ed. original: «La garantie jurisdictionnelle de la Constitution (La justice constitutionnelle)», Revue du Droit Publique et de la Science Politique en France et à l'étranger, XXXV].

$\Uparrow \quad$ ¿Quién debe ser el defensor de la Constitución? trad. de R.J. Brie (Madrid: Tecnos, 1925)

\section{KLIEMT, HARTMUT:}

$\Uparrow \quad$ 'Acerca de la coherencia de un liberalismo individualista estricto'en Ernesto Garzón Valdés (ed.), Derecho y filosofía, trad. de C. De Santiago (Barcelona: Alfa, 1985)

LANE, JAN-ERIK:

$\Uparrow \quad$ Constitutions and Political Theory (Manchester/New York: Manchester University Press, 1996)

\section{LAPORTA, FRANCISCO J:}

$\Uparrow \quad$ 'Sobre el concepto de derechos humanos' en Doxa, 4 (1987), 23-46.

$\Uparrow \quad$ 'Acotaciones del trampolín: Respuesta a Roberto Vernengo' en Doxa, 7 (1990)

LASLETT, PETER y FISHKIN, JAMES:

$\Uparrow \quad$ Philosophy, Politics and Society. 5th series (Oxford: Blackwell, 1979)

LIJPHART, AREND:

$\Uparrow \quad$ Democracies (New Haven, Yale University Press, 1984)

\section{LINCOLN, ABRAHAM:}

$\Uparrow \quad$ "First Inaugural Address (March 4, 1861)", en Don E. Fehrenbacher (ed.), Abraham Lincoln: Speeches and Writings, 1859-1865 (New York, The Library of America, ([1861] 1989) 
LYONS, DAVID:

$\Uparrow \quad$ 'Rights, Claimants, and Beneficiaries' en American Philosophical Quarterly, 6 (1969)

MACCORMICK, NEIL:

$\Uparrow \quad$ 'Legal Rights' en P.M.S. Hacker y J. Raz (eds.), Law, Morality, and Society. Essays in Honour of H.L.A. Hart (Oxford: Oxford University Press, 1978)

MACINTYRE, ALASDAIR:

$\Uparrow \quad$ After Virtue (Notre Dame: University of Notre Dame, 1984)

MACKIE, J.L.:

$\Uparrow \quad$ Ethics. Inventing Right and Wrong (Harmondsworth: Penguin, 1977)

$\Uparrow \quad$ 'Can There Be A Right-Based Moral Theory?' en Jeremy Waldron (ed.), Theories of Rights (Oxford: Oxford University Press, 1984)

MARX, KARL:

$\Uparrow \quad$ 'On the Jewish Question' en Karl Marx: Selected Writings, David McLellan (ed.) (Oxford: Oxford University Press, 1977)

MAY, KENNETH:

$\Uparrow \quad$ "A Set of Independent Necessary and Sufficient Conditions for Simple Majority Decision", Econometrica, 20 (1952) 680-684.

MILL, JOHN STUART:

$\Uparrow \quad$ Utilitarianism en Collected Works of John Stuart Mill, vol 10, J.M Robson (ed.) (Toronto: University of Toronto Press, 1969)

MILLER, NICHOLAS R.:

$\Uparrow \quad$ 'Majority Rule and Minority Interests', en I. Shapiro y R. Hardin (eds.), 
Political Order. Nomos XXXVIII (New York/Londres: New York University Press, 1996)

MORESO, JOSÉ JUAN:

^ La teoría del Derecho de Bentham (Barcelona: P.P.U., 1992)

$\Uparrow \quad$ La indeterminación del Derecho y la interpretación de la Constitución (Madrid: Centro de Estudios Políticos y Constitucionales, 1997)

NELSON, WILLIAM N.:

$\Uparrow \quad$ On Justifying Democracy (Londres, Routledge \& Kegan Paul, 1980)

NINO, CARLOS S.:

^ Etica y Derechos Humanos Un ensayo de fundamentación (Barcelona: Ariel, 1989)

^ 'La filosofía del control judicial de constitucionalidad' en Revista del Centro de Estudios Constitucionales, 4 (1989)

$\Uparrow \quad$ 'Sobre los derechos morales' en Doxa, 7(1990)

$\Uparrow \quad$ Fundamentos de Derecho Constitucional (Buenos Aires: Astrea, 1992)

PARFIT, DEREK:

$\Uparrow \quad$ Reasons and Persons (Oxford: Oxford University Press, 1984)

PARKER, RICHARD D.:

$\Uparrow \quad$ "Here, the People Rule". A Constitutional Populist Manifesto (Cambridge, Mass./London, Harvard University Press, 1994)

\section{RAE, DOUGLAS W.:}

$\Uparrow \quad$ 'Decision Rules and Individual Values in Constitutional Choice', American Political Science Review, 63 (1969) 40-56 
RAWLS, JOHN:

$\Uparrow \quad$ A Theory of Justice (Cambridge, Mass.: Harvard University Press, 1971)

$\Uparrow \quad$ Political Liberalism (New York: Columbia University Press, 1993)

RAZ, JOSEPH:

$\Uparrow \quad$ The Morality of Freedom (Oxford: Oxford University Press, 1986)

$\Uparrow \quad$ 'Rights and Politics' en Indiana Law Journal, 71 (1995)

ROSS, ALF:

$\Uparrow \quad$ On Law and Justice (Londres, Stevens \& Sons, 1958)

SANDEL, MICHAEL:

$\Uparrow \quad$ Liberalism and the Limits of Justice (Cambridge, Cambridge University Press, 1982)

SCHAUER, FREDERICK:

$\Uparrow \quad$ 'Judicial Review of the Devices of Democracy', Columbia Law Review, 94 (1994) 1326-1347

SCHELLING, THOMAS C:

$\Uparrow \quad$ 'Ethics, Law and the Exercise of Self-Command', en S. McMurrin (ed.), The Tanner Lectures on Human Values: vol. IV (Salt Lake City: University Of Utah Press/Cambridge, Cambridge University Press, 1983)

$\Uparrow \quad$ 'Ethics, Law and the Exercise of Self-Command' en Choice and Consequence. Perspectives of An Errant Economist (Cambridge, Mass.; Harvard University Press, 1984)

SEN, AMARTYA:

$\Uparrow \quad$ 'The Impossibility of Paretian Liberal' en Journal of Political Economy, 78 (1970) Collective Choice and Social Welfare (San Francisco: Holden-Day, 1979) 
SUMNER, L. W.:

$\Uparrow \quad$ The Moral Foundation of Rights (Oxford: Oxford University Press, 1987)

SUNSTEIN, CASS R.:

$\Uparrow \quad$ The Partial Constitution (Cambridge, Mass./Londres: Harvard University Press, 1993)

TAYLOR, CHARLES:

$\Uparrow \quad$ Sources of the Self (Cambridge: Cambridge University Press, 1989)

\section{TRIBE, LAURENCE H.:}

$\Uparrow \quad$ 'The Puzzling Persistence of Process-Based Constitutional Theories' en Yale Law Journal, 89 (1980)

\section{VERNENGO, ROBERTO J:}

$\Uparrow \quad$ 'Los derechos humanos como razones morales justificatorias' en Doxa, 7 (1990)

\section{WALDRON, JEREMY:}

$\Uparrow \quad$ Theories of Rights, Jeremy Waldrom, editor (Oxford: Oxford University Press, 1984)

$\Uparrow \quad$ Nonsense upon Stilts. Bentham, Burke and Marx of the Rights of Man, Jeremy Waldrom editor (Londres: Methuen, 1987)

$\Uparrow \quad$ 'A Right-Based Critique of Constitutional Rights', Oxford Journal of Legal Studies, 13 (1993) 18-51.

$\Uparrow \quad$ 'Freeman's Defense of Judicial Review', Law and Philosophy, 13 (1994) 27-41.

$\Uparrow \quad$ 'Vagueness in Law and Language: Some Philosophical Issues' en California Law Review, 82 (1994)

$\Uparrow \quad$ 'Legislation, Authority and Voting', Georgetown Law Journal, 84 (1996) 21852214. 
WALZER, MICHAEL:

$\Uparrow \quad$ Spheres of Justice (New York: Basic Books, 1983)

WILLIAMS, BERNARD:

$\Uparrow \quad$ Ethics and the Limits of Philosophy (Londres, Fontana Press, 1985)

ZAGREBELSKY, GUSTAVO:

$\Uparrow \quad$ I/ diritto mite: legge, diritti, giustizia (Turín: Einaudi, 1992) 